\title{
Establishment of nude mouse transplantable model of a human adenoid cystic carcinoma of the oral floor showing metastasis to the lymph node and lung
}

\author{
SUSUMU HASHITANI, MASAHIRO URADE, YUSUKE ZUSHI, \\ EMI SEGAWA, SHIN OKUI and KAZUNARI SAKURAI \\ Department of Oral and Maxillofacial Surgery, Hyogo College of Medicine, \\ 1-1 Mukogawa-cho, Nishinomiya, Hyogo 663-8501, Japan
}

Received July 11, 2006; Accepted September 27, 2006

\begin{abstract}
Adenoid cystic carcinoma (ACC) is a generally slow-growing but highly malignant salivary gland neoplasm with remarkable capacities for local invasion and lung metastasis. The precise characteristics of ACC are not fully understood because there was no suitable animal model. We have successfully established a new human tumor line (ACCI) derived from ACC of the oral floor, which showed a cribriform pattern histologically and serially transplantable into nude mice. This tumor developed spontaneous metastasis to the neck at the second passage level, and the histological feature changed from ACC to undifferentiated carcinoma (ACCIM). ACCIM caused spontaneous metastasis to the lung at high incidence when transplanted subcutaneously in nude mice. In this study, we examined the characteristics of this interesting human ACC metastatic line. Tumor fragments were subcutaneously transplanted into nude mice and tumor growth was measured at 1-week intervals. Histological and immunohistochemical examinations were performed. As a result, the tumor growth rate of ACCIM increased as compared to that of ACCI, and the PCNA labeling index was elevated. Furthermore, ACCIM produced multiple metastases to lymph nodes and lungs 5 months after transplantation, and all mice died within 6 months. These multiple metastases were also confirmed in orthotopic transplantation to the tongue. RT-PCR analysis revealed that ACCIM expressed human $\beta$-actin, indicating its human origin. From these findings, ACCIM transplanted into nude mice would provide a useful model for investigating the biological behaviour of ACC.
\end{abstract}

Correspondence to: Dr Susumu Hashitani, Department of Oral and Maxillofacial Surgery, Hyogo College of Medicine, 1-1 Mukogawa-cho, Nishinomiya, Hyogo 663-8501, Japan

E-mail: susumu@hyo-med.ac.jp

Key words: adenoid cystic carcinoma, oral floor, metastatic model, nude mice

\section{Introduction}

Adenoid cystic carcinoma (ACC) is a generally slow-growing but highly malignant salivary gland neoplasm with remarkable capacities for invasion and metastasis. Patients with conventional ACC of the salivary glands show a fair 5-year survival rate, but have a poor clinical outcome after prolonged observation $(10-20$ years $)(1,2)$. The long-term overall survival of these patients is poor, owing to the occurrence of late recurrences or metastases, even for low-grade tumors $(1,2)$. Approximately $40-60 \%$ of patients develop distant metastases (lungs, bones, and soft tissues), despite good local control of the tumor (3). Although this tumor shows a unique biological behavior, the precise characteristics are not fully understood. One of the reasons is that there were no suitable cell lines or animal models of ACC to analyze. Only two studies including ours have recently reported a heterotransplantation model of human ACC into nude mice $(4,5)$.

We have successfully established a new human tumor line derived from ACC of the oral floor, which showed a cribriform pattern histologically and serially transplantable into nude mice. This tumor developed spontaneous metastasis to the neck at the second passage level, and the histological feature changed from ACC to undifferentiated carcinoma. This metastatic tumor caused spontaneous metastasis to the lung at high incidence when transplanted in nude mice.

In this study, we report the establishment and characterization of this new interesting human ACC metastatic line.

\section{Materials and methods}

Animals. Five-week-old female nude mice (BALB/c nu/nu; Oriental Yeast Ltd., Tokyo, Japan) were used for the animal experiment, and fed ad libitum under sterile conditions. All animal experiments were performed in compliance with the Guidelines for Animal Experiments of Hyogo College of Medicine.

Tumor tissue and heterotransplantation into nude mice. The original tumor tissue was obtained from operated material of a 72-year-old male patient with ACC of the oral floor showing a cribriform pattern. The metastatic tumor tissue was obtained 


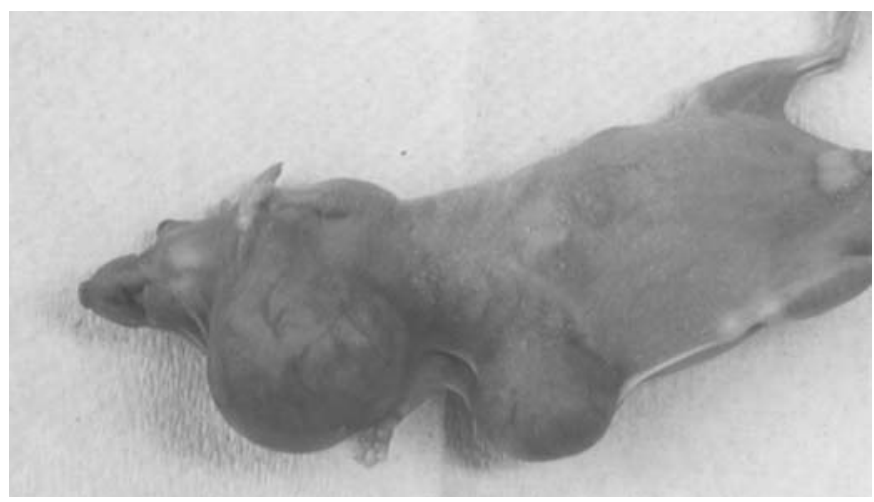

Figure 1. Photomicrographs of ACCI at second passage and initial ACCIM in a nude mouse.

from a lesion of spontaneous neck metastasis of the above ACC occurring at the second passage level in a nude mouse (Fig. 1). After being rinsed 3 times in phosphate-buffered saline (PBS), the tumor was cut into approximately $2 \times 2-\mathrm{mm}$ pieces and transplanted into the flanks of the mice. The length (L) and width $(\mathrm{W})$ of the tumor formed at the transplanted site were measured once a week, and the relative tumor weight was calculated as $\mathrm{W}^{2} \mathrm{xLx} 1 / 2$, according to the method of Battelle Columbus Laboratories (6). Tumors were routinely transferred every two months into nude mice.

Some of the tumor was also transplanted orthotopically into the dorsal portion of the tongue.
Histological and immunohistochemical examinations. The tissue specimens were fixed in $10 \%$ formalin, embedded in paraffin, cut into $4-\mu \mathrm{m}$ sections and stained with hematoxylin and eosin (H\&E) for histological examination.

Immunohistochemical examination was performed by using an avidin-biotin-peroxidase complex (ABC) staining method (7). Briefly, the specimens were blocked for endogenous peroxidase activity by treatment with $3 \% \mathrm{H}_{2} \mathrm{O}_{2}$ in methanol for $5 \mathrm{~min}$. After washing with PBS, they were treated with $1 \%$ normal horse serum in PBS for $15 \mathrm{~min}$ and then were incubated with monoclonal antibody against PCNA (Biogenex, San Ramon, CA, USA). After further washing with PBS, specimens were incubated with the ABC complex solution (Vectastain, Vector Lab., Burlingame, CA, USA) at room temperature for $15 \mathrm{~min}$ and biotinylated goat anti-mouse IgG (Vector) was applied to the sections, which were then incubated for $30 \mathrm{~min}$ at room temperature. The specimens were treated with a substrate solution which contained 3,3'diaminobenzidine tetrahydrochloride (Wako Pure Chemical Industries, Ltd., Osaka, Japan) and $\mathrm{H}_{2} \mathrm{O}_{2}$ for approximately 5 min. Finally, the specimens were counterstained with hematoxylin, dehydrated and mounted with glycerol gelatin. The PCNA labeling index (PCNA L.I.) was obtained by counting the ratio of PCNA-positive cells to total 1,000 tumor cells in the well-labeled areas determined by scanning at low magnification.

RNA preparation. Total RNA was isolated from exponentially growing cells using Isogen (Nippon Gene Co., Tokyo, Japan),
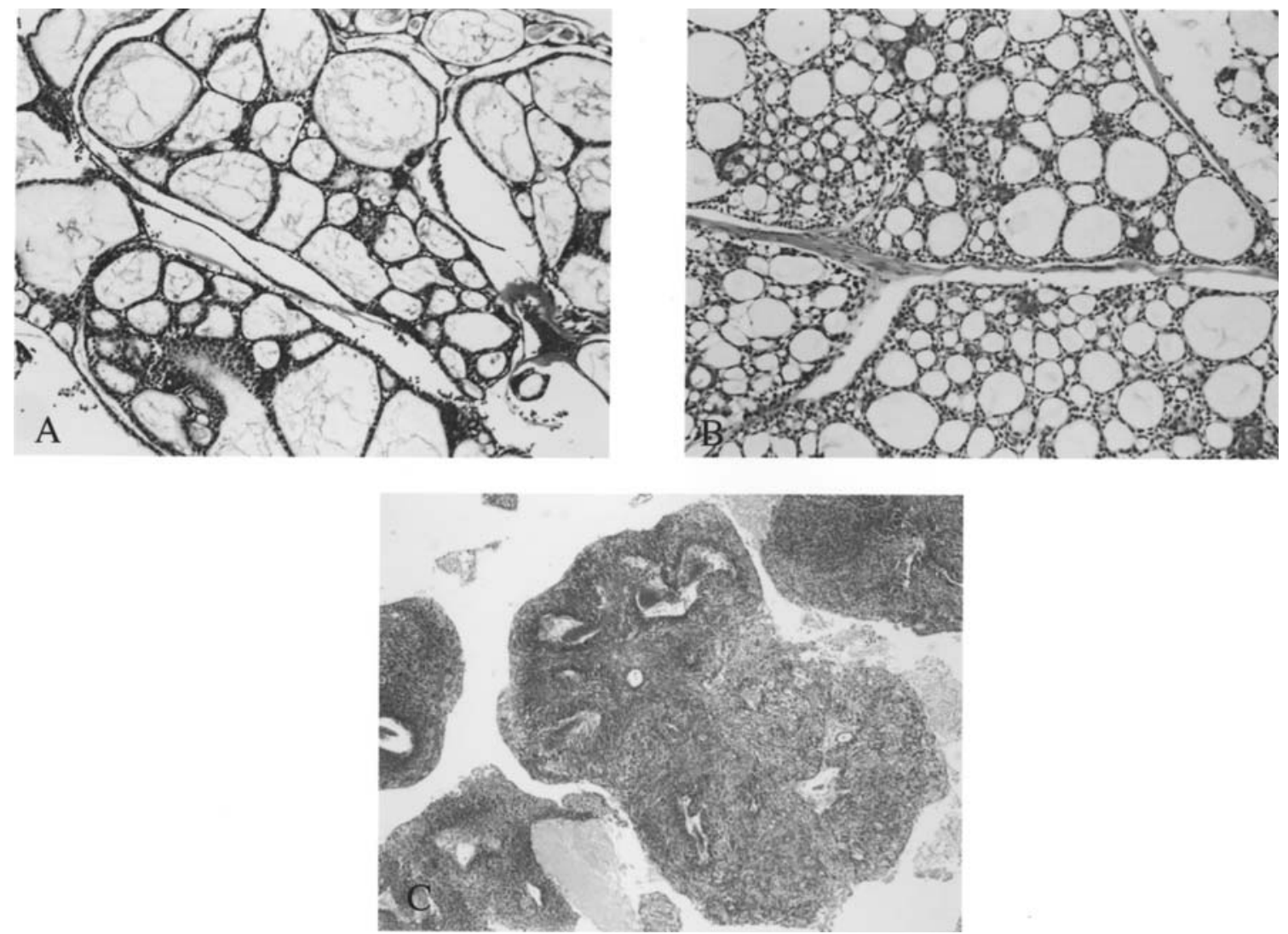

Figure 2. Photomicrographs of the histology of ACC. (A) primary ACC occurring in the oral floor of the patient showing a cribriform pattern; (B) ACCI tumor in nude mice; and (C) ACCIM tumor in nude mice (H\&E; original magnification, $\mathrm{x} 100)$. 
A

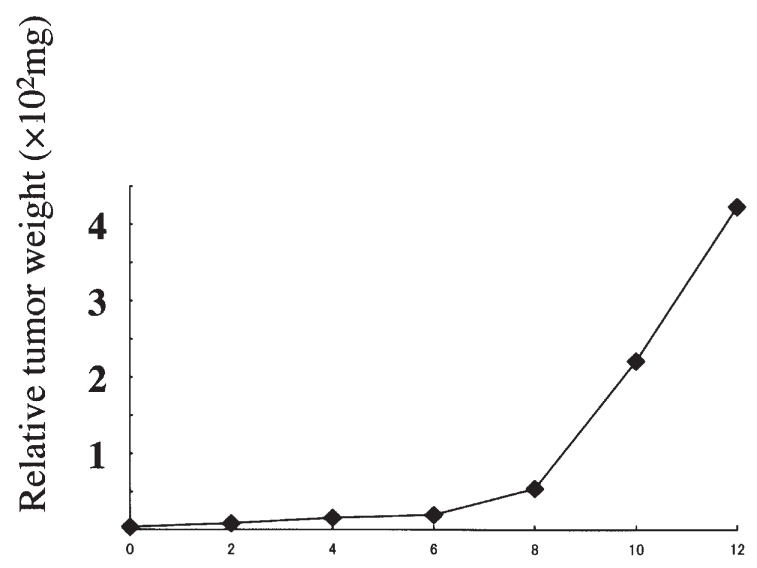

Months
B

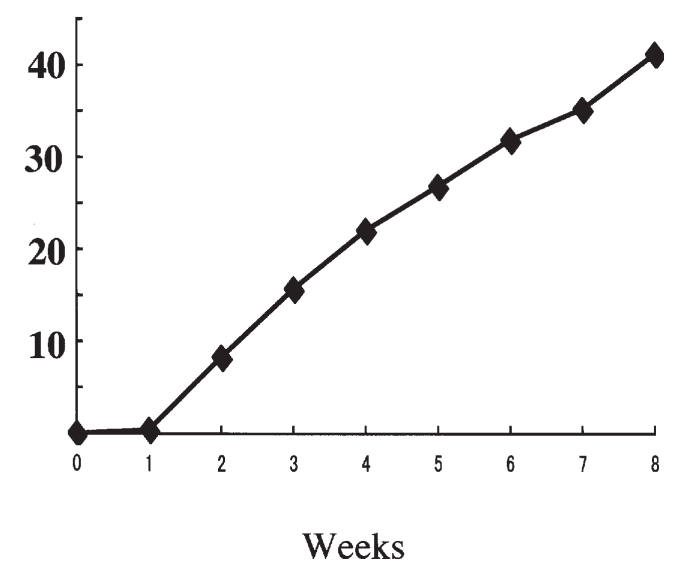

Weeks

Figure 3. Tumor growth curve of the ACCI (A) and ACCIM (B) in nude mice.
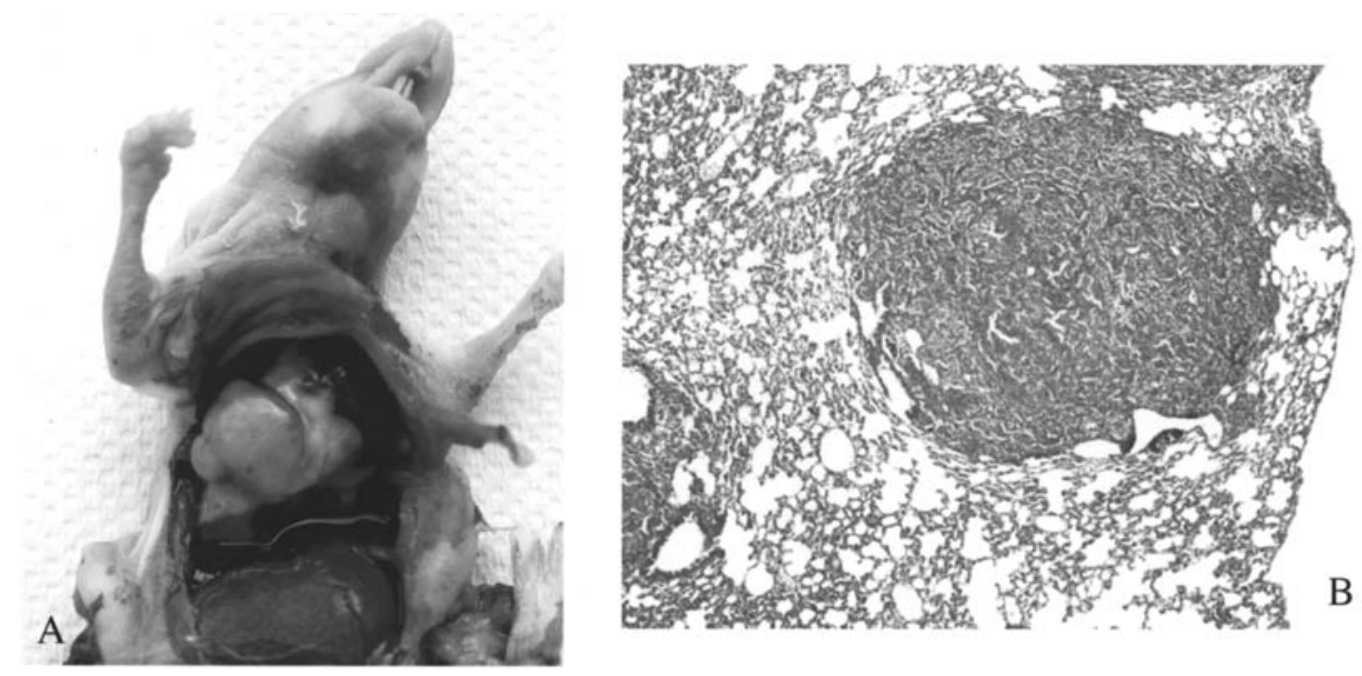

Figure 4. Lung metastasis in nude mouse by ACCIM serial passages (A) and histology (B) (H\&E; original magnification, x40).

treated with RNase-free DNase (Promega Co., Tokyo) at $37^{\circ} \mathrm{C}$ for $10 \mathrm{~min}$ and then extracted with phenol-chloroform.

Reverse transcription-polymerase chain reaction ( $R T-P C R)$ analysis. This ACC metastatic tumor was evaluated detecting the PCR product for the human and mouse B-actin genes to prove that it originates from human tissue but not from mouse tissue. The SCC25 cell line derived from squamous cell carcinoma of the tongue was used as the human control (8). PCR of human $\beta$-actin cDNA was conducted using a sense primer, 5'-TGACGGGGTCACCCACACTGTGCCCATCTA3', and an antisense primer, 5'-CTAGAAGCATTTGCGGTG GACGATGGAGGG-3', at $94^{\circ} \mathrm{C}$ for $5 \mathrm{~min}$, then 35 cycles at $94^{\circ} \mathrm{C}$ for $30 \mathrm{sec}, 66^{\circ} \mathrm{C}$ for $30 \mathrm{sec}$, and $72^{\circ} \mathrm{C}$ for $1 \mathrm{~min}$, and then at $72^{\circ} \mathrm{C}$ for $7 \mathrm{~min}$. The primers for mouse $\beta$-actin were sense, 5'-GATGACGATATCGCTGCGCTG-3', and antisense, 5'GTACGACCAGAGGCATACAGG-3', at $94^{\circ} \mathrm{C}$ for $5 \mathrm{~min}$, then 35 cycles at $94^{\circ} \mathrm{C}$ for $30 \mathrm{sec}, 58^{\circ} \mathrm{C}$ for $1 \mathrm{~min}$, and $72^{\circ} \mathrm{C}$ for $1 \mathrm{~min}$, and then at $72^{\circ} \mathrm{C}$ for $7 \mathrm{~min}$. PCR products were electrophoresed in agarose gels, stained with ethidium bromide and visualized under UV illumination.

\section{Results}

Transplantation of the ACC tumor and metastatic ACC tumor into nude mice and metastasis to the lung. When small pieces of the operated material from ACC of the oral floor were transplanted into nude mice, tumors developed at the transplanted site two months later and grew slowly. Histologically, transplanted ACC tumor was demonstrated as ACC identical to the original tumor of the patient (Fig. 2A and B). As the tumors were transplanted serially as ACCI, tumor growth became rapid gradually.

At the second passage level, approximately 1 year after ACCI transplantation, another tumor mass appeared in the neck apart from the transplanted site of the tissue fragment (Fig. 1). 

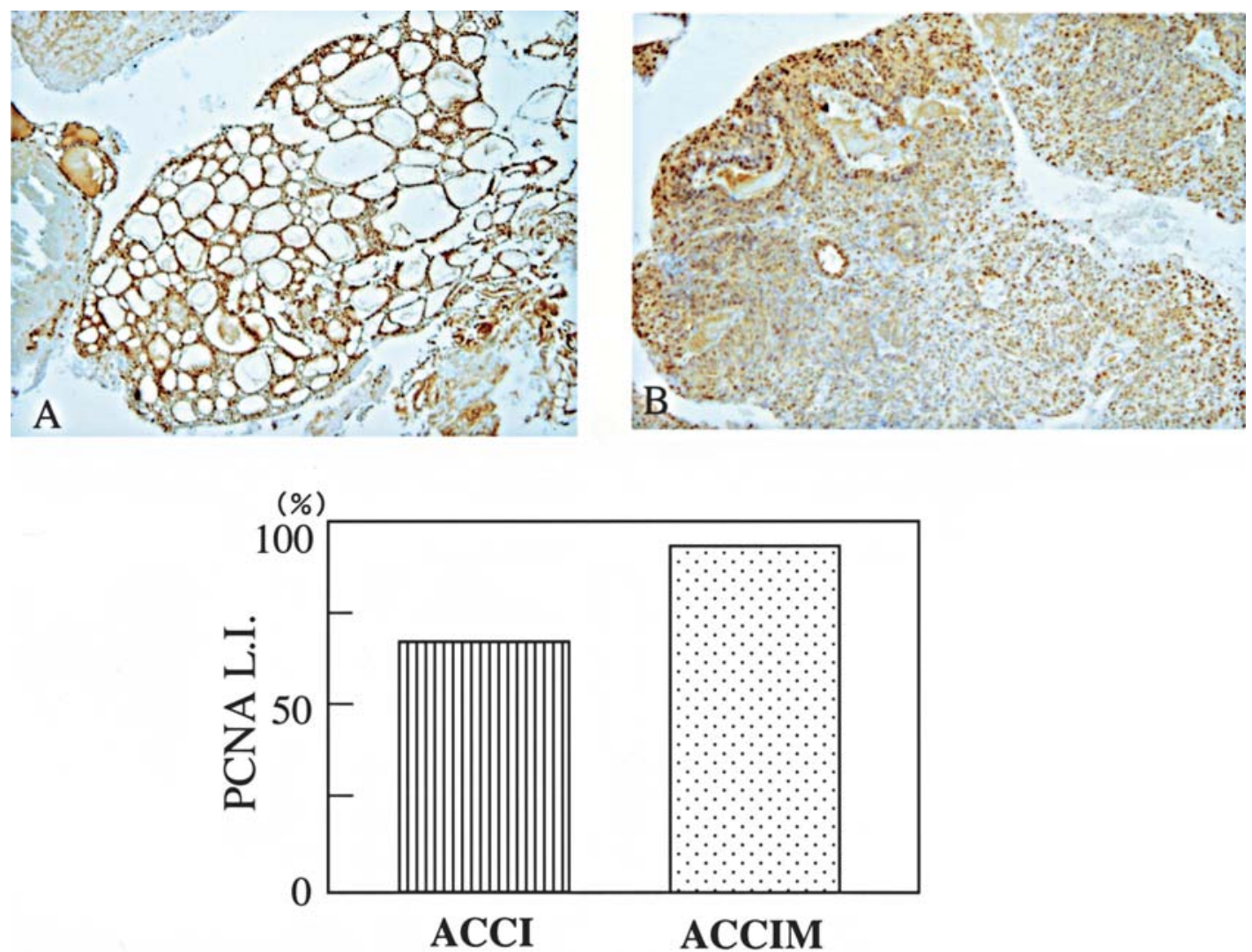

Figure 5. Immunohistochemistry of PCNA stain. ACCI (A) and ACCIM (B) (original magnification, x100), and labeling index.
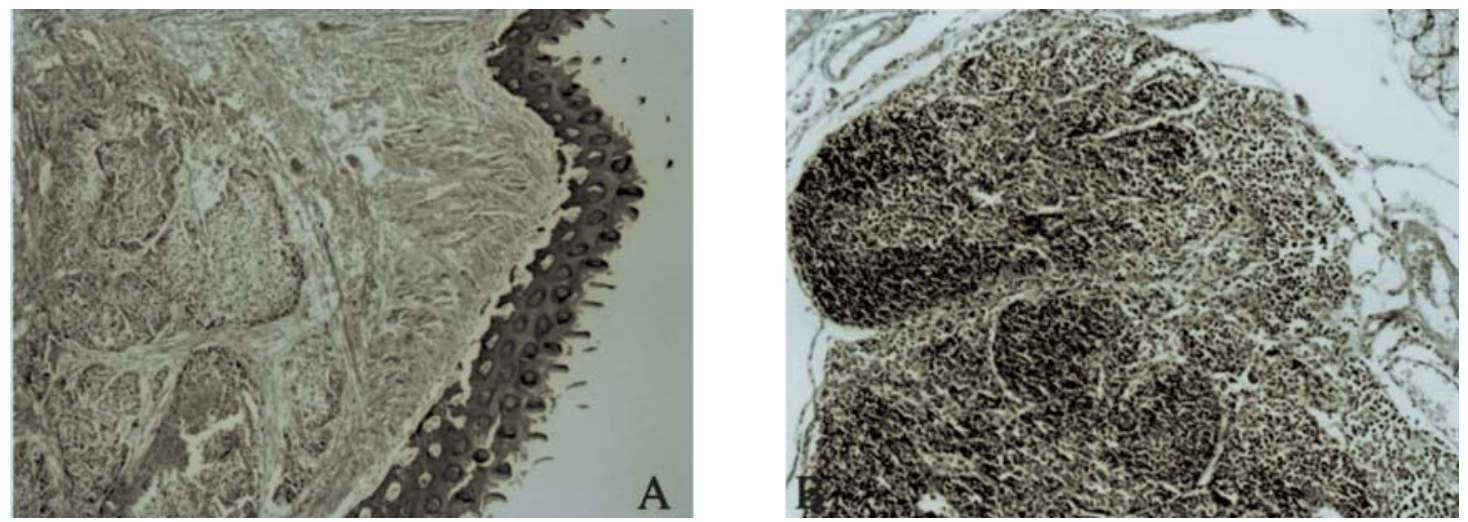

Figure 6. The ACCIM transplanted orthotopically into the tongue (A), and metastasis to the lymph node (B) in a nude mouse (H\&E; original magnification, $\mathrm{x} 100)$.

Although this tumor showed an undifferentiated carcinoma histologically, it was considered as a metastatic lesion and designated ACCIM (Fig. 2C). ACCIM demonstrated very rapid growth and tumor weight was approximately 500-fold larger than that of the ACCI at 2 months post-transplantation (Fig. 3A and B). In addition, almost all nude mice transplanted with ACCIM showed multiple lung metastases approximately 5 months after transplantation in the flanks (Fig. 4A and B). The metastases were also observed in axillary lymph nodes, liver and spleen as far as examined.

Immunohistochemical analysis for PCNA. The immunohistochemical examination revealed an increase of PCNA staining in ACCIM tumor cells as compared with that in ACCI. The

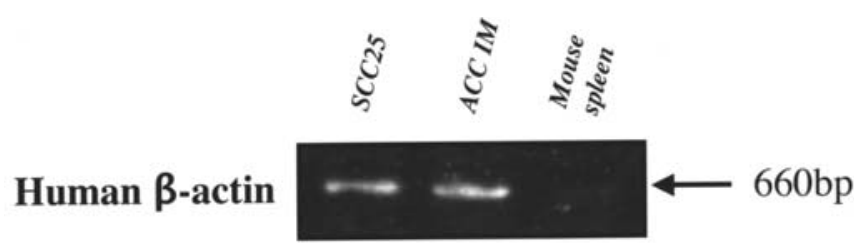

Mouse $\beta$-actin

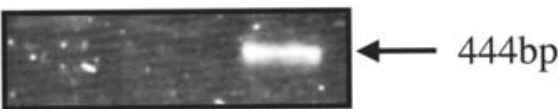

Figure 7. RT-PCR analysis of B-actin of human and mouse in the ACCIM tumor. 
immunoreactivity for PCNA was detected in the cell nuclei, and PCNA L.I. was $70.2 \%$ at the ACCI and $92.3 \%$ at the ACCIM (Fig. 5A and B).

Metastases to the lymph node and the lung by orthotopic transplantation. The ACCIM was orthotopically transplanted into the tongue of nude mice with a success rate of $50 \%$ $(5 / 10)$. Histologic examinations revealed an undifferentiated carcinoma similar to the ACCIM, and invasive proliferation without capsule formation (Fig. 6A). Three out of five mice with the tongue tumor showed histological evidence of lymph node metastases (Fig. 6B) to the submandibular and axillary lymph nodes and to the lung.

PCR analysis for human $\beta$-actin. Using RT-PCR analysis to elucidate the tissue origin of ACCIM, we examined the expression levels of human and mouse $B$-actin. As a result, the ACCIM tumor and SCC25 cell line derived from human tongue carcinoma demonstrated a band of human $\beta$-actin, but not mouse $\beta$-actin. In contrast, the mouse $\beta$-actin band was detected in a mouse spleen extract (Fig. 7).

\section{Discussion}

In an attempt to investigate the biological characteristics of ACC, several authors have established some ACC cell lines through tissue culture (9-11). These cell lines originated from ACC of the cribriform type, but failed to produce tumors by subcutaneous transplantation into nude mice. We have previously established an ACC tumor serially transplantable into nude mice, and demonstrated that this tumor originated from ACC with a cribriform pattern, but changed to a solid pattern as the tumor growth rate increased after serial passages (5). Umeda et al also reported an ACC tumor with a solid pattern that metastasized to the lungs (4). As far as we know, no ACC tumor with a cribriform pattern and serially transplanted into nude mice has been established so far. This ACCI has successfully been passaged for 71 months and is now at the 7th passage level. The initially heterotransplanted ACC line had histologically the same cribriform pattern of the original ACC excised from the patient.

Cancer's metastatic process involves a cascade of linked, sequential steps involving multiple host-tumor interactions. To create successfully a metastatic deposit, a cell, or group of cells, must be able to leave the primary tumor, invade the local host tissue, enter the circulation, arrest at the distant vascular bed, extravasate into the target organ interstitium and parenchyma, and proliferate as a secondary colony (12). Many attempts to establish an in vivo experimental model with a high rate of metastases have been reported. These are divided into 2 categories, in vitro and in vivo selection methods, and they are useful to elucidate the precise metastatic mechanism, but they do not allow the reproduction of local infiltration and vascular invasion of the metastatic initial stage. These methods are suitable for a similar metastatic model for a clinical tumor that transplants a cancer cell in animal subcutis to reproduce a process of cancer metastasis. It is desirable for a spontaneous metastasis model to spread, but it is rare for nude mice tumor xenografts to cause local infiltration and metastasis generally. We applied a model of spontaneous metastases and obtained an ACCI tumor subline, designated as ACCIM. Histologically, ACCIM is an undifferentiated carcinoma. Umeda et al also reported an ACC that produced spontaneous metastases in nude mice with the features of an undifferentiated carcinoma (13). The ACCIM tumor is also serially transplantable into nude mice subcutaneous tissue, and spontaneously metastasizes to the lungs and axillary lymph nodes of nude mice. Lung metastases are detectable by routine histological examinations 5 months after transplantation. It is reported that the reason for the development of spontaneous metastases in the lungs is that this metastatic tumor retains the property of ACC to easily metastasize (4). It has been reported that, in contrast with a heterotopically transplanted tumor, an orthotopically transplanted tumor was capable of invading local tissues and metastasizing $(14,15)$. When the ACCIM tumor was transplanted to the tongues of nude mice, it invaded and metastasized to the lymph nodes, as well as to the lungs.

RT-PCR was used to confirm that ACCIM was not of murine origin. As the PCR product of the human B-actin gene was detected and that of the mouse $B$-actin was not detected in the ACCIM tumor, it was confirmed to be of human origin. These findings suggested that ACCIM was a metastatic lesion from ACCI.

The establishment of the ACCI and ACCIM cell line is now under investigation. This ACCIM tumor derived from ACC, which is transplantable into nude mice, might serve as a useful metastatic model for investigating the biological behavior of ACC.

\section{Acknowledgments}

This study was supported by a Grant-in-Aid for Scientific Research from the Ministry of Education, Culture, Sport, Science and Technology of Japan to S.H. (no. 17791503) and M.U. (no. 18390549), and the Hyogo College of Medicine Research Funds.

\section{References}

1. de Kerviler E, Bely N, Laccourreye O, Clement O, Halimi P and Frija G: The aryepiglottic fold as a rare location of adenoid cystic carcinoma. Am J Neuroradiol 16: 1375-1377, 1995.

2. Fordice J, Kershaw C, El-Naggar A and Goepfert H: Adenoid cystic carcinoma of the head and neck. Predictors of morbidity and mortality. Arch Otolaryngol Head Neck Surg 125: 149-152, 1999.

3. Ellis GL and Auclair PL: Tumors of the salivary glands. Atlas of tumor pathology. 3rd edition. Armed Forces Institute of Pathology, Bethesda (MD), pp203-216, 1996.

4. Umeda M, Komatsubara H, Nishimatsu N, et al: Establishment and characterization of a human adenoid cystic carcinoma line of the salivary gland which is serially transplantable and spontaneously metastasizes to the lung in nude mice. Oral Oncol 38: 30-34, 2002.

5. Hashitani S, Noguchi K, Manno Y, et al: Changes of histological and biological features by serial passages in a human adenoid cystic carcinoma line transplantable in nude mice. Oncol Rep 13: 607-612, 2005.

6. Ovejera AA, Houchens DP and Baker AD: Chemotherapy of human tumor xenografts in genetically athymic mice. Ann Clin Lab Sci 8: 50-56, 1978.

7. Hsu SM, Raine L and Franger H: Use of avidin-biotin-peroxidase complex $(\mathrm{ABC})$ in immunoperoxidase techniques: a comparison between $\mathrm{ABC}$ and unlabeled antibody (PAP) procedures. J Histochem Cytochem 29: 577-580, 1981 .

8. Rheinwald JG and Beckett MA: Tumorigenic keratinocyte lines requiring anchorage and fibroblast support cultures from human squamous cell carcinomas. Cancer Res 41: 1657-1663, 1981. 
9. He RG, Zhang XS, Zhou XJ, et al: The establishment of cell lines of adenoid cystic carcinoma of human salivary glands (ACC2, ACC3) and a study of morphology. West Chin J Stomatol 6: 1-4, 1988.

10. Sobue M, Takeuchi J, Niwa M, et al: Establishment of a cell line producing basement membrane components from an adenoid cystic carcinoma of the human salivary gland. Virchows Arch B Cell Pathol Include Mol Pathol 57: 203-208, 1989.

11. Shirasuna K, Saka M, Hayashido Y, Yoshioka H, Sugiura T and Matsuya T: Extracellular matrix production and degradation by adenoid cystic carcinoma cells: participation of plasminogen activator and its inhibitor in matrix degradation. Cancer Res 53: 147-152, 1993.

12. Liotta LA, Steeg PS and Stetler-Stevenson WG: Cancer metastasis and angiogenesis: an imbalance of positive and negative regulation. Cell 64: 327-336, 1991.
13. Umeda M, Komatsubara H, Nishimatsu N, et al: Establishment and characterization of metastasizing cell lines from heterotransplanted human adenoid cystic carcinoma. Oral Surg Oral Med Oral Pathol Oral Radiol Endod 98: 211-216, 2004.

14. Kawashiri S, Kumagai S, Kojima K, Harada H and Yamamoto E: Development of a new invasion and metastasis model of human oral squamous cell carcinomas. Eur J Cancer B Oral Oncol 31: 216-221, 1995.

15. Nakajima M, Morikawa K, Fabra A, Bucana CD and Fidler IJ: Influence of organ environment on extracellular matrix degradative activity and metastasis of human colon carcinoma cells. J Natl Cancer Inst 82: 1890-1898, 1990. 